\title{
Lighting Studies For Fuelling Machine Deployed Visual Inspection Tool
}

Carl Stoots, George Griffith

April 2015

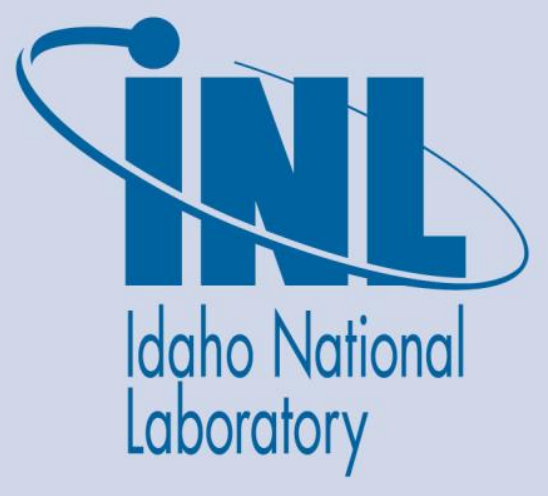

The INL is a U.S. Department of Energy National Laboratory operated by Battelle Energy Alliance 
INL/EXT-15-35074

\title{
Lighting Studies For Fuelling Machine Deployed Visual Inspection Tool
}

\author{
Carl Stoots, George Griffith
}

April 2015

Idaho National Laboratory
Idaho Falls, Idaho 83415

http://www.inl.gov

Prepared for the U.S. Department of Energy

Under DOE Idaho Operations Office

Contract DE-AC07-05ID14517 


\section{SUMMARY}

Under subcontract to James Fisher Nuclear, Ltd., INL has been reviewing advanced vision systems for inspection of graphite in high radiation, high temperature, and high pressure environments. Earlier calculations and measurements performed by INL indicated that a remote telescopic inspection might be feasible [1]. In this concept, a vision tube (similar to a periscope) optically couples the interrogation region (surface features of fuel channels within graphite blocks in the reactor core) with a still or video camera above the core. The vision tube provides a clear optical path approximately $20 \mathrm{~m}$ long, the diameter of which is constrained by the geometry of the EDF UK Fueling Machine Inspection Tool. If the vision tool were the sole measurement technique of the FMIT, vision tube diameters of up to $100 \mathrm{~mm}$ might be possible. More likely compatible vision tube diameters are $30-50 \mathrm{~mm}$. AMEC conducted proof-ofprinciple testing of a integrated vision tube: camera, telescopic lens, lighting, conical mirror, and image processing [2]. One of AMEC's findings was that significantly more developmental effort needed to be done on appropriate lighting techniques. INL conducted a review of AMEC's efforts [3] and continued earlier efforts by studying two potential remote (i.e., co-located with the camera and telescopic lens) lighting techniques: a laser diode array and a focused beam LED light.

INL calculations and measurements, as well as consideration of AMEC measurement results, lead to the following tentative conclusions. It appears promising that a $43 \mathrm{~mm}$ diameter, $400 \mathrm{ml}$ telescopic lens with a rather low cost CMOSbased digital camera will detect $0.5 \mathrm{~mm}$ width surface features on certain graphite substrates at $20 \mathrm{~m}$ offset distance using a remote (co-located with the telescopic lens) focused beam LED lighting system of about 1000 lumens. It seems that the concave fuel channel interior surface of non-irradiated AGR graphite is compatible with this inspection system whereas other types of graphite surfaces may not be as easily interrogated with this inspection concept. Furthermore, the choice of $43 \mathrm{~mm}$ diameter telescopic optics should fit in the geometric limitations of the FMIT as it is currently understood. Finally, the power requirements of the camera and focused beam LED lighting system and should be compatible with the existing wiring in the FM. The particular camera used by INL is USB-based for data transmission (and power). The FMIT should probably use a Ethernet-based camera for data transmission, due to lead lengths and data transmission signal timing. 


\section{Table of Contents}

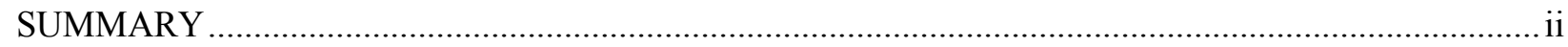

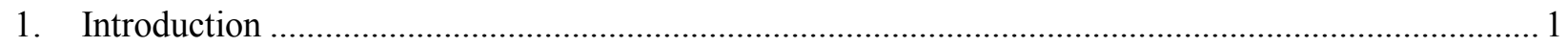

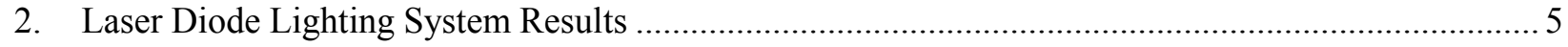

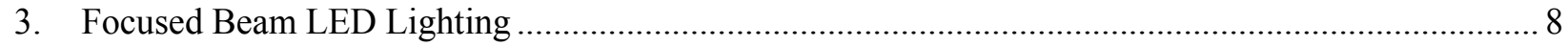

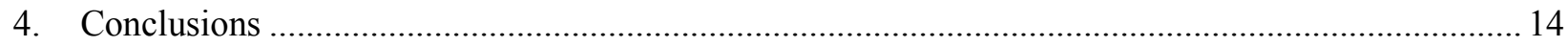

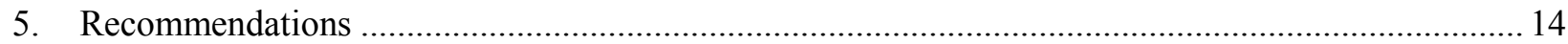

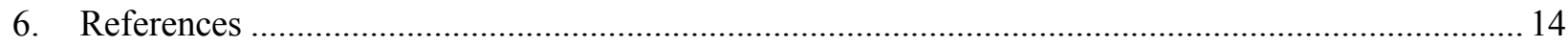




\section{Lighting Studies for Fuelling Machine Deployed Visual Inspection Tool}

\section{Introduction}

Under subcontract to James Fisher Nuclear, Ltd., INL has been reviewing advanced vision systems for inspection of graphite in high radiation, high temperature, and high pressure environments. As Advanced Gas Reactor (AGR) cores age, more frequent inspection of core graphite blocks is required to ascertain integrity, cracking, dimensional changes, etc. Current technology requires that the reactor be depowered, depressurized, and partially cooled. Fuel rods are removed from targeted channels, and moved out of the way using the AGR Fuelling Machine. A separate device called the Channel Bore Inspection Equipment is inserted above the empty fuel channel, and inspection equipment lowered into the graphite core. The fuel rod shuffling required for this inspection technique significantly prolongs shutdowns, making inspections costly. It is desired to integrate a visual inspection instrument into a Fuelling Machine Inspection Tool (FMIT) used by the Fuelling Machine (FM), greatly reducing inspection time and cost.

The simplest radiation resistant way to obtain visual access of the graphite core integrity would be to mount a digital camera and all optics at the top of the FMIT (outside the highest radiation and temperature environment) and provide an unobstructed optical path down the length of the FMIT. This concept is like a telescope looking down into the core. A sealed tube (either evacuated or backfilled with appropriate gas) would provide a clear uninterrupted optical path approximately $20 \mathrm{~m}$ long, the diameter of which is constrained by the geometry of the FMIT and FM itself. The inspection tip would include a right hand mirror / conical mirror to collect light reflected from the graphite surface and direct the light upwards within the tube to the telescopic lens / camera arrangement mounted above.

There has been much discussion about what maximum tube diameter could be accommodated by the FMIT, given that the FMIT would probably not be dedicated solely to visual inspection of graphite blocks (channel diameter is $127 \mathrm{~mm}$ ). This maximum diameter is important for many reasons: diameter and length together dictate the minimum angular separation between two targets that can be distinguished (diffraction limit), and tube diameter dictates lens diameter and hence light gathering area. Furthermore, any impact the FMIT may have on the FM is important as well. At the EDF / Ristek Fuelling Machine Core Inpsection Tool Stakeholder \& Supplier Workshop held November 20, 2013, several boundaries were established for the FMIT any measurements to be incorporated into the FMIT. Measurements being considered include channel bore, channel tilt, bulk eddy current, crack detection via grab cable load measurement, visual inspection, and detailed eddy current inspection. EDF's vision of the MK1 FMIT includes a bottom brush with load cell to detect gross cracks, 3 and/or 5 circumferential eddy current cells, LVDT feelers for bore measurements, brushes for tilt measurements, and vision tube for visual inspection. The consensus at the supplier workshop was that $30-50 \mathrm{~mm}$ tube diameter was reasonable.

Both INL and AMEC has studied the viability of this concept. INL has made calculations to determine the theoretical performance of such a concept, and made early experimental measurements using a $400 \mathrm{~mm}$ focal length telescopic lens and high sensitivity CMOS camera (Orion Starshoot Autoguider monochrome, $5.2 \mu \mathrm{m}$ pixel size). AMEC further developed the concept by incorporating a conical mirror, image unwrapping and image stitching software, and specialized lighting techniques.

Early on, INL performed some simple calculations to define the optical resolution of various telescopic lens diameters and focal lengths. The resolution for a CCD-based imaging system is given by:

$$
R_{C C D}(\text { arcsec } / \text { pixel })=\frac{\text { pixel size }[\mu \mathrm{m}]}{\text { focal length }[\mathrm{mm}]} * 206.3
$$

The spatial scale can be calculated from the angular resolution via:

$$
\text { Image Scale }(\mathrm{mm} / \text { pixel })=2 * 1000 * L * \tan \left(\frac{R_{C C D}}{2}\right)
$$


where $\mathrm{L}$ is the distance to the target in meters.

If one assumes a possible unobstructed optical path down the length of the FMIT of up to 4" diameter, typical optical focal lengths would range up to about $1000 \mathrm{~mm}$. Table 1 lists the resulting CCD image scale for various optical focal lengths for a typical imaging camera with $5.2 \mu \mathrm{m}$ pixel size.

Table 1. Image scale for different optical focal lengths.

\begin{tabular}{|c|c|c|c|}
\hline $\begin{array}{c}\text { Focal Length } \\
(\mathrm{mm})\end{array}$ & $\begin{array}{c}\text { CCD Image Scale } \\
(\text { arcsec/pixel })\end{array}$ & $\begin{array}{c}\text { CCD Image Scale } \\
\text { at 10m (mm/pixel) }\end{array}$ & $\begin{array}{c}\text { CCD Image Scale } \\
\text { At 20 m (mm/pixel) }\end{array}$ \\
\hline 200 & 5.4 & 0.26 & 0.52 \\
\hline 300 & 3.6 & 0.17 & 0.35 \\
\hline 400 & 2.7 & 0.13 & 0.26 \\
\hline 500 & 2.1 & 0.10 & 0.21 \\
\hline 600 & 1.8 & 0.09 & 0.17 \\
\hline 700 & 1.5 & 0.07 & 0.15 \\
\hline 800 & 1.3 & 0.07 & 0.13 \\
\hline 900 & 1.2 & 0.06 & 0.12 \\
\hline 1000 & 1.1 & 0.05 & 0.10 \\
\hline
\end{tabular}

Table 1 indicates that from an image scale argument, it is possible to resolve a $1 \mathrm{~mm}$ crack from a distance of $10 \mathrm{~m}$ or 20 $\mathrm{m}$ with a typical camera and optical configuration. Also, the image scale for a given camera pixel size is only a function of optical focal length and is independent of optical aperture.

However, there is a theoretical minimum angular separation between two sources that can be distinguished by a telescope arrangement. This theoretical minimum (diffraction limit) is dependent only upon the diameter of the optics (optical aperture) and is independent of camera or focal length. The diffraction limit is given by:

$$
R(\text { radians })=\frac{1.22 \lambda}{D}
$$

where $\mathrm{R}$ is the angular diffraction limit, $\lambda$ is the wavelength of light (assume green light $550 \times 10^{-9} \mathrm{~m}$ ), and $\mathrm{D}$ is the aperture (telescope diameter). So, for a $50 \mathrm{~mm}$ diameter telescope, the diffraction limit is $1.342 \times 10^{-5}$ radians. Table 2 presents calculations of the diffraction limit for different telescope apertures for two distances: $10 \mathrm{~m}$ and $20 \mathrm{~m}$.

Table 2. Diffraction limit (theoretically highest optical resolution) for different lens apertures.

\begin{tabular}{|c|c|c|}
\hline Aperture (mm) & Diffraction Limit $(\mathrm{mm})$ at $10 \mathrm{~m}$ & Diffraction Limit $(\mathrm{mm})$ at $20 \mathrm{~m}$ \\
\hline 43 & 0.156 & 0.31 \\
\hline 50 & 0.134 & 0.27 \\
\hline 60 & 0.112 & 0.22 \\
\hline 70 & 0.096 & 0.20 \\
\hline 80 & 0.084 & 0.17 \\
\hline 90 & 0.075 & 0.15 \\
\hline 100 & 0.067 & 0.13 \\
\hline
\end{tabular}

It is useful to interpret these calculations for the two different optical arrangements used by INL and AMEC. INL's optical measurements and image acquisition tests concentrated upon a $43 \mathrm{~mm}$ diameter, $400 \mathrm{~mm}$ focal length (f9.3) telescopic lens combined with a high sensitivity $1280 \times 1024$ pixel, 8 bit A/D, $5.2 \mu$ m pixel size monochrome CCD camera. AMEC conducted optical measurements with a $100 \mathrm{~mm}$ diameter, $800 \mathrm{~mm}$ focal length (f8) telescopic lens combined with a 2208 x 3000 pixel, 10 bit A/D, $3.5 \mu \mathrm{m}$ pixel size color CMOS camera (PixeLink PL-A782). Note that the smaller pixel size of the camera employed by AMEC is offset by it being a color camera with Bayer matrix (repeated $2 \times 2$ array of pixels made up of 2 greens, 1 blue and 1 red to assemble a RGB image), and hence the effective pixel size is 
larger. The effective spatial resolution of such one-shot color cameras is dependent upon the specific mathematical debayering and inter-pixel interpolation algorithm used by the camera. For the purposes of discussion here, we will assume the effective pixel sizes are the same between the two cameras.

The above calculations indicate that:

- $\quad$ assuming no optical aberrations due to imperfect optics (spherical aberration, coma, astigmatism, curvature, etc.)

- $\quad$ ignoring the effects of index of refraction variations along the optical path

- assuming proper illumination

the theoretically highest optical resolution (diffraction limit) for a $43 \mathrm{~mm}$ diameter telescope, $400 \mathrm{~mm}$ focal length, at 20 $\mathrm{m}$ distance is $0.31 \mathrm{~mm}$. A $100 \mathrm{~mm}$ lens can theoretically discern details as small as $0.13 \mathrm{~mm}$ in size. The image scales for these two optical systems are $0.26 \mathrm{~mm} / \mathrm{pixel}$ and $0.13 \mathrm{~mm} / \mathrm{pixel}$, respectively. In other words, each system given the above assumptions should discern surface features less than $0.5 \mathrm{~mm}$ in size. EDF has specified that detection of cracks of at least $0.5 \mathrm{~mm}$ in width is a minimum specification of any visual inspection system. The conclusion is that for a reasonable optical path diameter $(\sim 50 \mathrm{~mm})$ the CCD camera and all lenses could be located in the FMIT above the core within the concrete containment area where temperatures and dose rates are significantly lower.

Image acquisition tests conducted by INL using pencil leads of $0.5 \mathrm{~mm}, 1 \mathrm{~mm}$, and $2 \mathrm{~mm}$ diameter against the backdrop of an actual AGR graphite block have shown that features of $0.5 \mathrm{~mm}$ or larger are indeed discernable using a $43 \mathrm{~mm}$ diameter lens at $18.4 \mathrm{~m}$ distance (maximum distance available in lab) with ambient lighting. Figure 1 shows the entire field of view using the $43 \mathrm{~mm}$ diameter lens at $18.4 \mathrm{~m}$ distance. Figure 2 is a digitally magnified crop of Figure 1 . The $0.5 \mathrm{~mm}$ diameter pencil lead is easily visible.

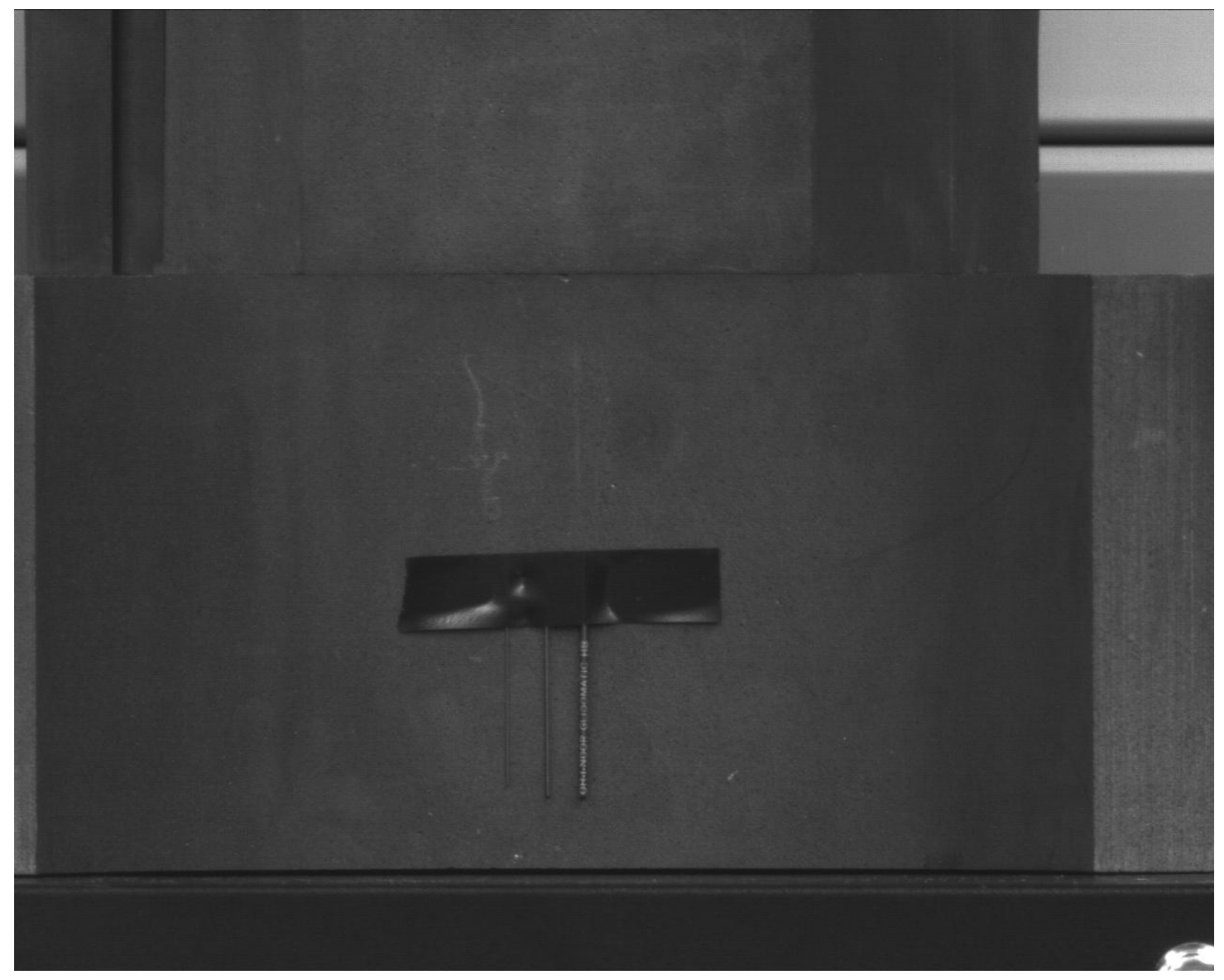

Figure 1. Image of graphite rods / blocks at 18.4 meters distance using $43 \mathrm{~mm}$ diameter optics (400 mm focal length). 

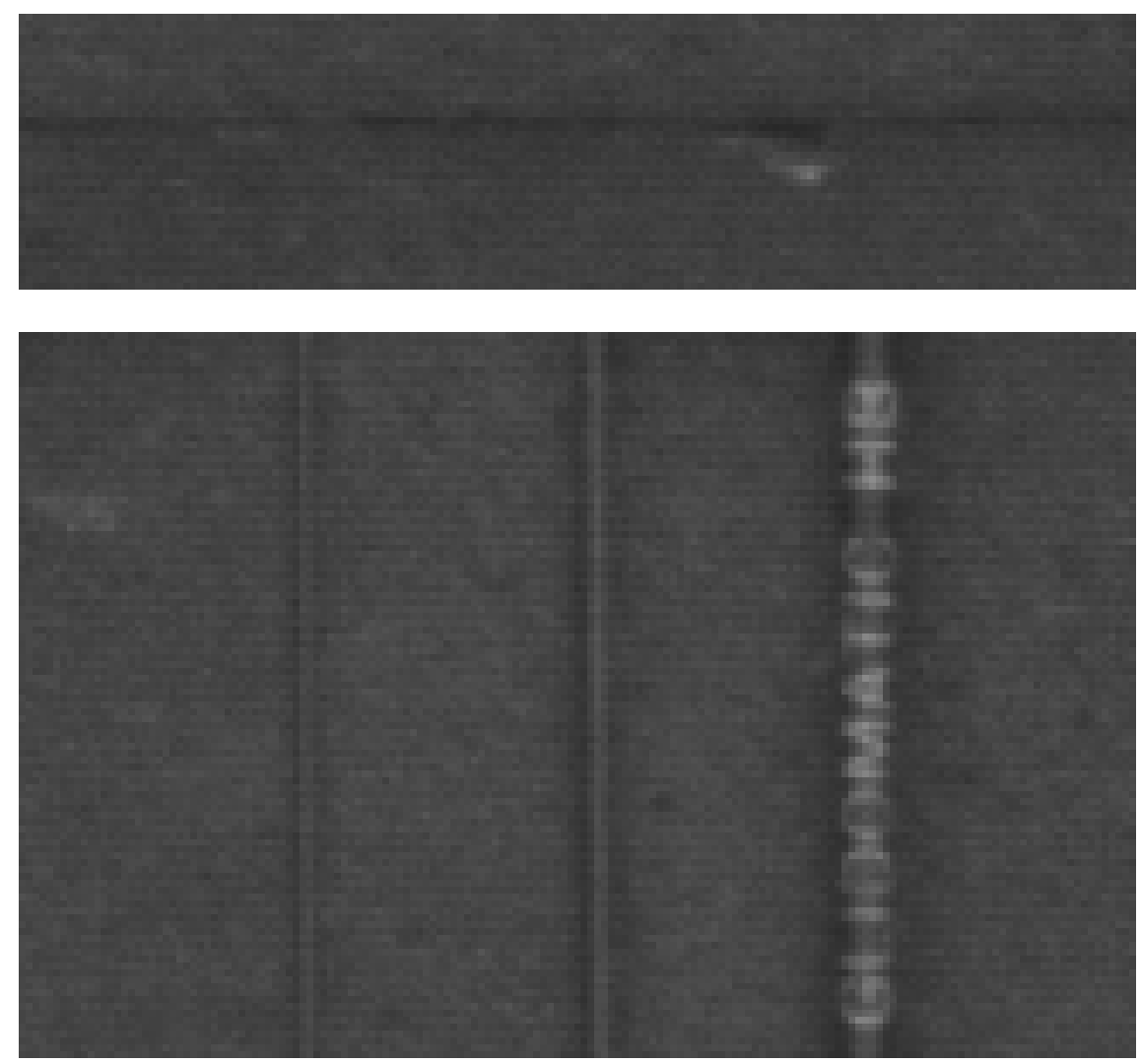

Figure 2. Two digitally magnified crops of Figure 4. Rods in the lower crop are 0.5 $\mathrm{mm}, 1 \mathrm{~mm}$ and $2 \mathrm{~mm}$ in diameter (left to right). Upper crop shows the interfacial gap between the upper and lower graphite block.

It is recognized, however, that proper illumination of the graphite block is key to any visual inspection technique. Not only must the lighting be of appropriate intensity, but should be compatible with the tool's operating environment (temperature, radiation, and maximum FMIT power availability of $80 \mathrm{~W}$ ). The lighting intensity requirement also depends upon lens diameter. For example, based upon aperture area and ignoring focal length, a $43 \mathrm{~mm}$ diameter lens requires $5.4 \mathrm{x}$ as much light as a $100 \mathrm{~mm}$ diameter lens. AMEC conducted some tests of a $110 \mathrm{~mm}$ diameter clear acrylic light guide. The guide was $150 \mathrm{~mm}$ long and utilized $24 \mathrm{LEDs}$ at one end to direct a total of 670 lumens to the interrogation region. From vendor literature for the LEDs utilized, total lighting power consumption for this system is approximately $19 \mathrm{~W}$. AMEC recognized that for this system to be practical in the FMIT, the light guide would need to be fabricated from non-browning glass and use optical fibers to guide the light from a source outside the core region. The maximum operating temperature for these LEDs is $85 \mathrm{C}$ and hence the LEDs would not survive the environment if inserted into the core. However, suggesting the use of optical fibers is not a viable solution. Most optical fibers will not survive the radiation exposure. Saphire fibers could survive the radiation exposure and temperature extremes, but will require too many fiber-to-fiber junctions (and corresponding light transmission losses) for 20m length.

The INL also explored lighting concepts compatible with the FMIT-based visual inspection tool. One was a laser-based lighting concept originally conceived for inspecting underground piping systems. The second concept explored by INL was a high intensity (1000 lumen) focused-beam LED lighting. Both these systems have the advantage of locating the lighting source at the top of the tool outside the high temperature, high radiation environment of the core without relying upon fiber optics or light guides. 


\section{Laser Diode Lighting System Results}

Figure 3 depicts the prototype laser diode system that was tested for applicability to graphite block illumination. The concept employs 10 laser diodes with integrated optics that form a 5x5 rectangular grid of light (Figure 4). In the radial center of the array of lasers is a removable camera. This camera was removed for testing so that the higher sensitivity Orion camera could be used.

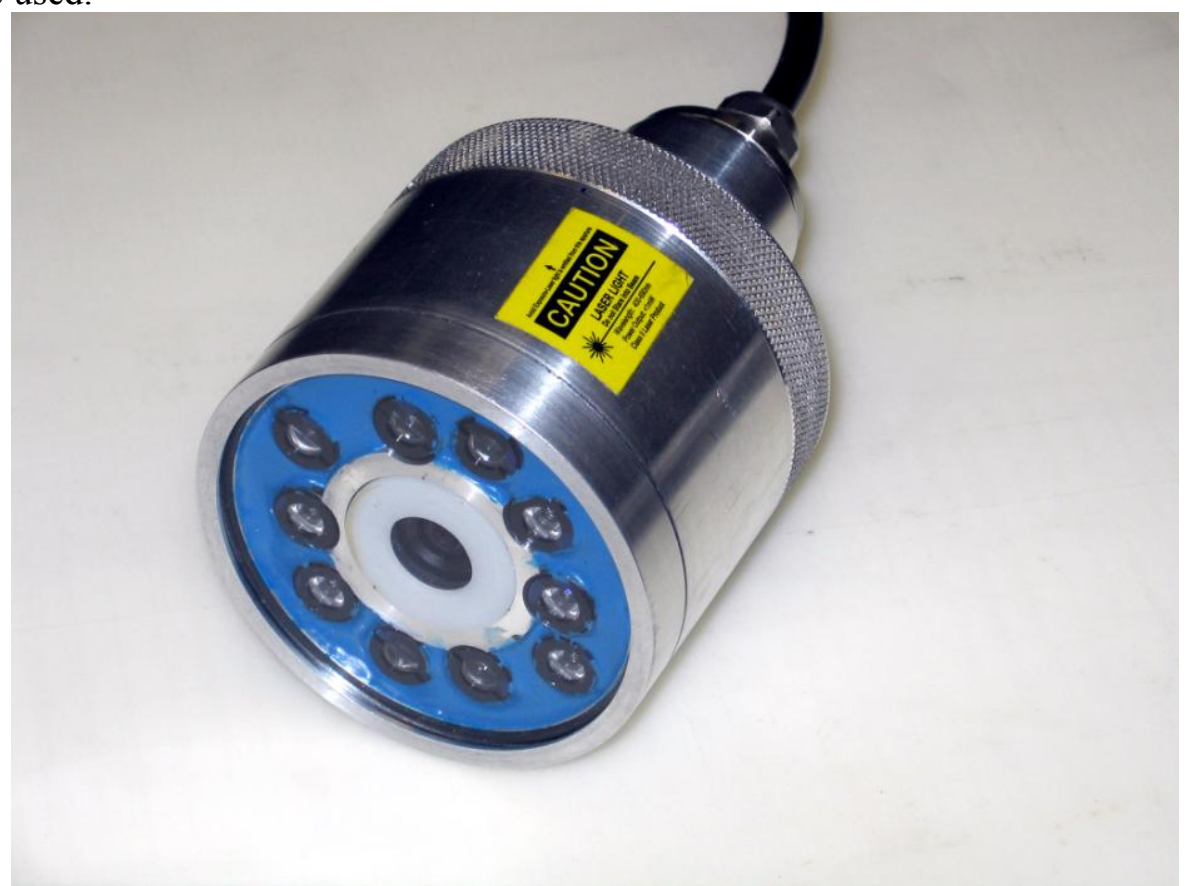

Figure 3. Co-linear laser diode system tested by INL for graphite block illumination.

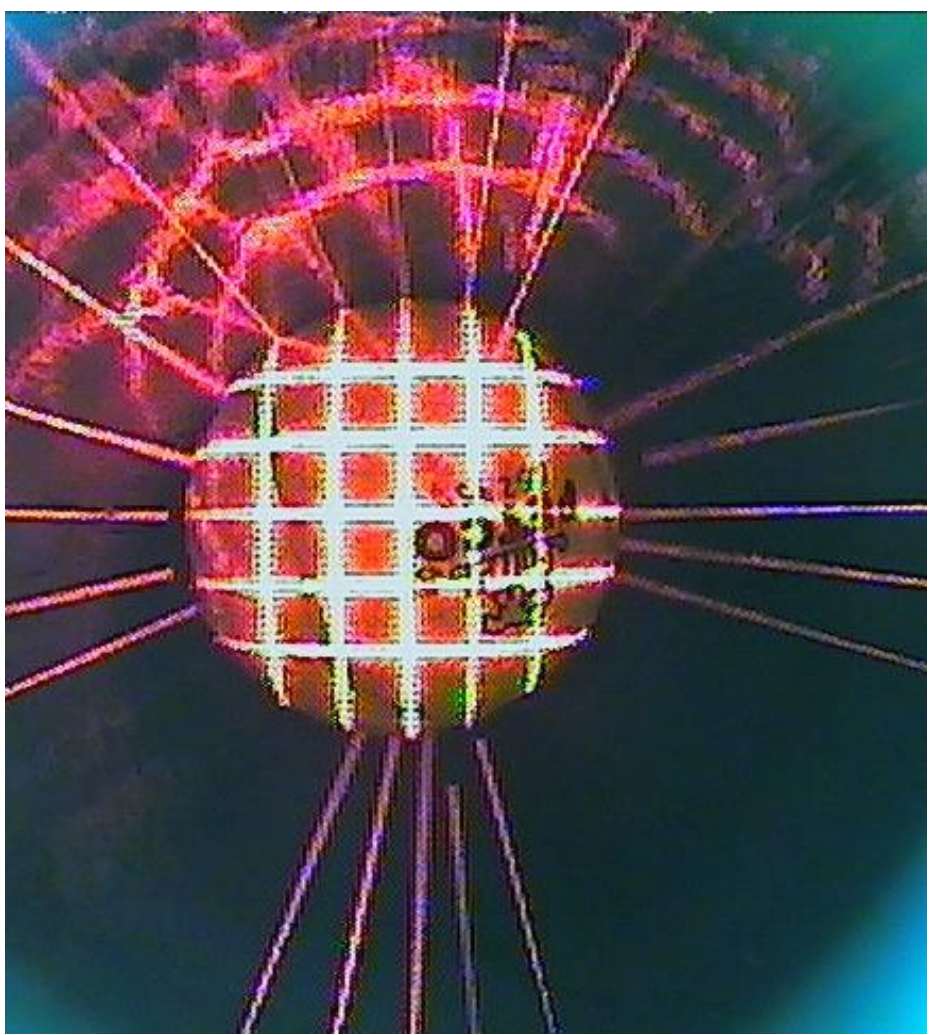

Figure 4. Grid pattern produced by laser diode system in Figure 3. 


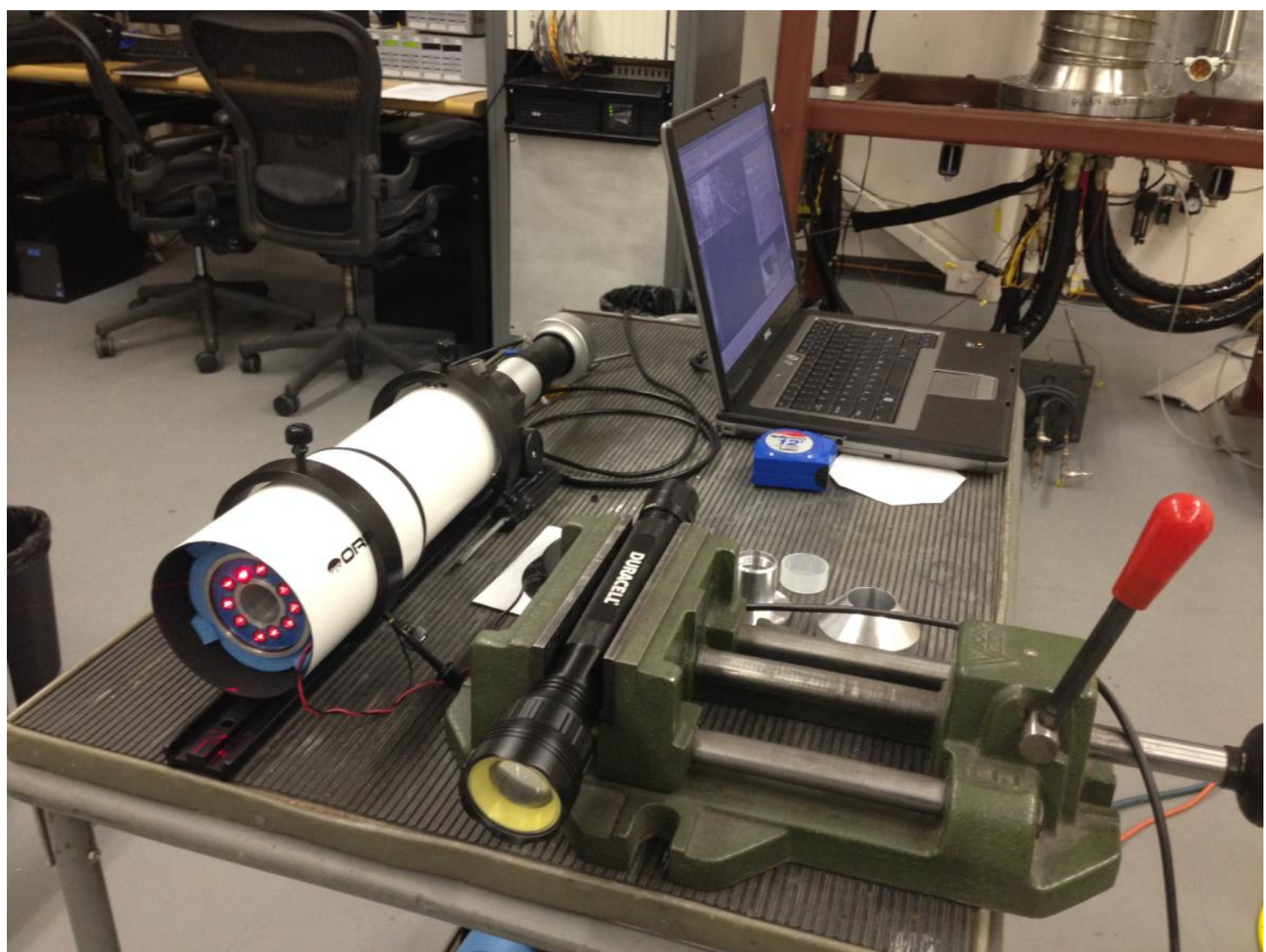

Figure 5. Laser diode system mounted within front of telescopic lens. Orion digital camera is mounted on rear of lens.

Figure 5 shows the laser diode optical configuration used. Total power consumption for the 10 laser diodes was approximately $5.2 \mathrm{~W}$. The central aperture for the laser diode optics was $25 \mathrm{~mm}$, which is significantly less than the 43 $\mathrm{mm}$ aperture previously used. This, of course, has significant implications for system sensitivity and resolution. However, for simple proof-of-principle testing purposes, it was deemed not necessary to redesign the existing configuration since the diffraction limit would still be approximately $0.5 \mathrm{~mm}$.

Figure 6 is a $10 \mathrm{~s}$ exposure image captured of a flat graphite block surface (upper half) and concave AGR graphite block channel (lower half). The laser diode optical configuration illustrated in Figure 5 was employed at a distance of $18.4 \mathrm{~m}$. No other lighting was utilized. Unfortunately, this lighting technique was quickly ruled out for two reasons.

First, the effectiveness of laser lighting on a graphite surface was strongly dependent upon the surface finish. Machined or sawn graphite surfaces tended to be very reflective while cast graphite surfaces tended to be very absorptive. It was difficult to predict how surface defects responded to laser illumination. Holes and cracks tended to be absorptive, which when superimposed on the cast graphite absorptive background, hampered any distinction or resolution. Surface scars from rubbing tended to be reflective.

Second, the laser light at $20 \mathrm{~m}$ distance had a very speckle pattern on the graphite surface. The speckles are an intensity pattern produced by mutual interference of the laser wavefronts. Speckle patterns are accentuated by diffuse reflections on materials such as paper, white paint, and other porous surfaces. The porous nature of cast graphite surfaces causes diffuse reflections and hence speckle patterns. Furthermore, any tiny vibrations in the laser optics are amplified over the $20 \mathrm{~m}$ path length, causing the speckle pattern to change with time (creating a dynamic speckle). Although speckle patterns can be used to advantage in certain types of interferometry, they are a severe drawback to using lasers to illuminate diffuse reflective materials. In the case of illuminating surface defects on graphite blocks, the speckles served to create overly grainy images with no detail. 


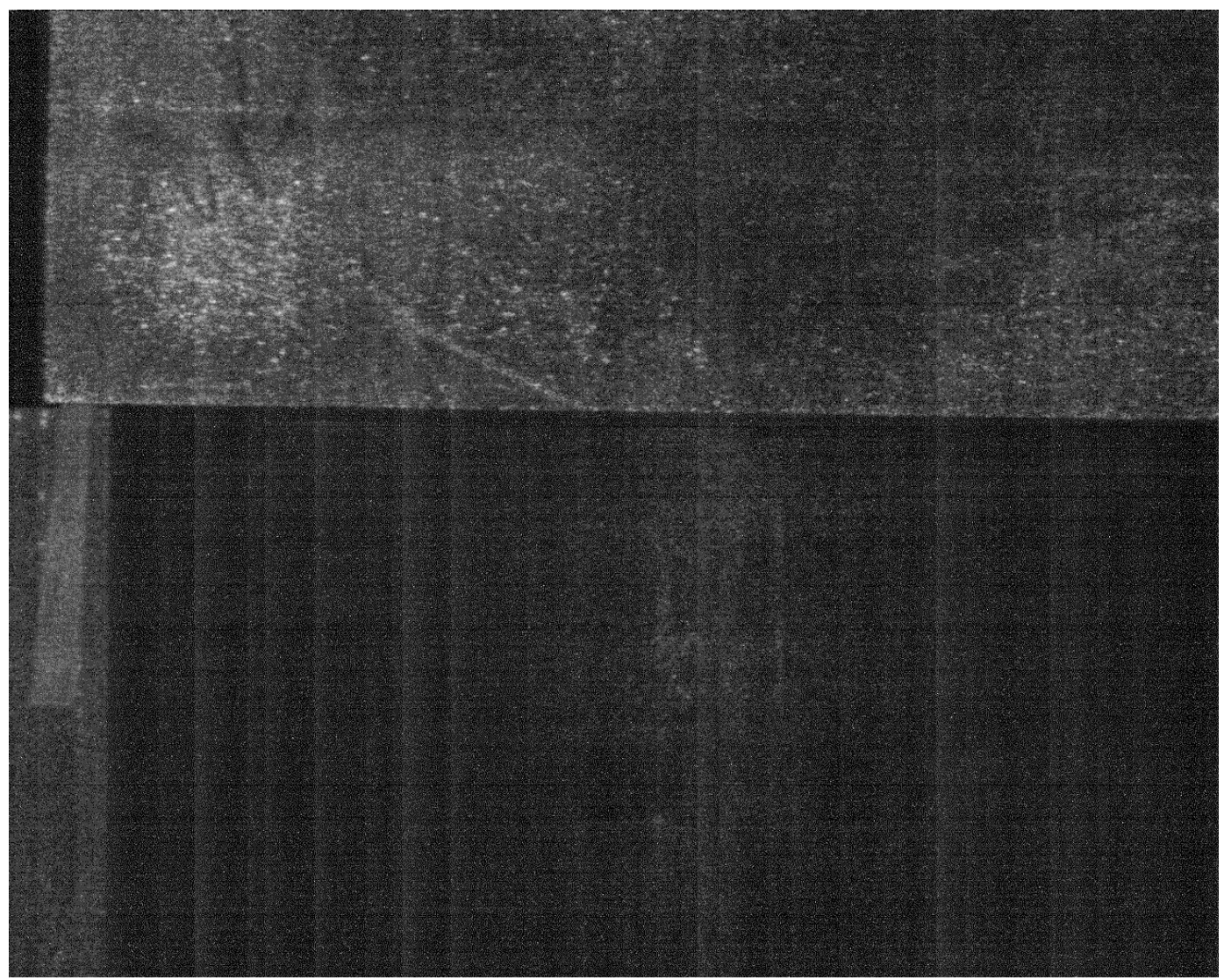

Figure 6. $10 \mathrm{sec}$ exposure of flat graphite block (upper) and concave AGR channel block (lower) using laser diode lighting.

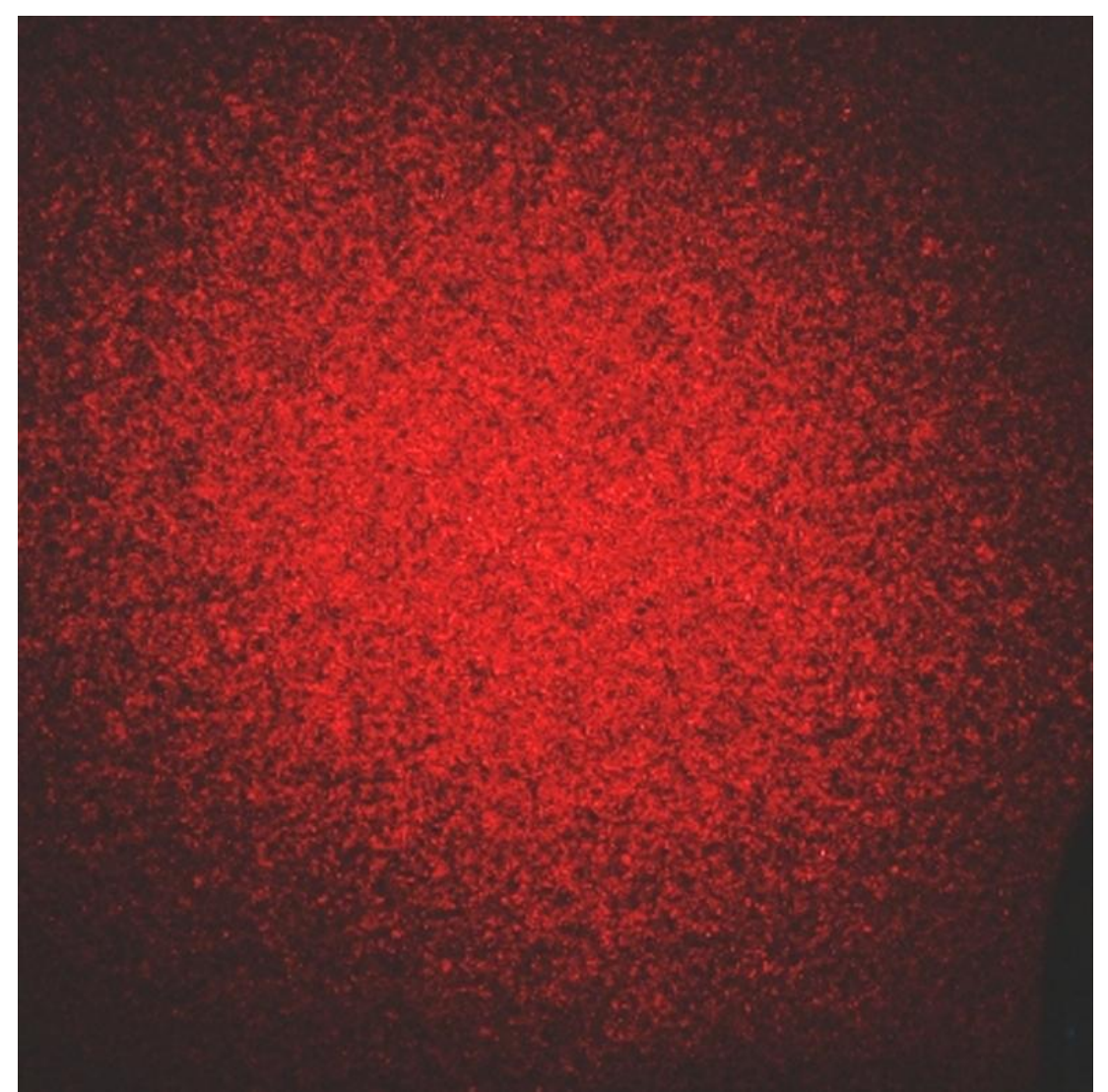

Figure 7. Photograph of a speckle pattern (Wikipedia, Speckle pattern). 


\section{Focused Beam LED Lighting}

The second remote lighting configuration explored by the INL was a focused beam high intensity LED light. A 1000 lumen flashlight (approximately $16 \mathrm{~W}$ electrical energy consumption) was focused at graphite targets $18.4 \mathrm{~m}$ distance. Figure 8 shows the lab bench setup with the focused bean LED light and telescopic receiving optics. The aperture of the receiving optics remained $43 \mathrm{~mm}$, and focal length $400 \mathrm{~mm}$. Figure 9 shows the focused LED beam spot size at $18.4 \mathrm{~m}$ distance. The AGR graphite block width is about $20 \%$ of the beam spot size width. Figure 10 displays the target used for initial scoping tests of the focused beam LED lighting concept. The target was comprised of an AGR graphite block with the concave hemispherical interior surface as a backdrop, with 3 pencil leads of $0.5 \mathrm{~mm}, 1.0 \mathrm{~mm}$, and $2.0 \mathrm{~mm}$ diameter, and several scratches / holes that were intentionally created on the graphite surface. These scratches and holes had widths / diameters of less than $0.5 \mathrm{~mm}$.

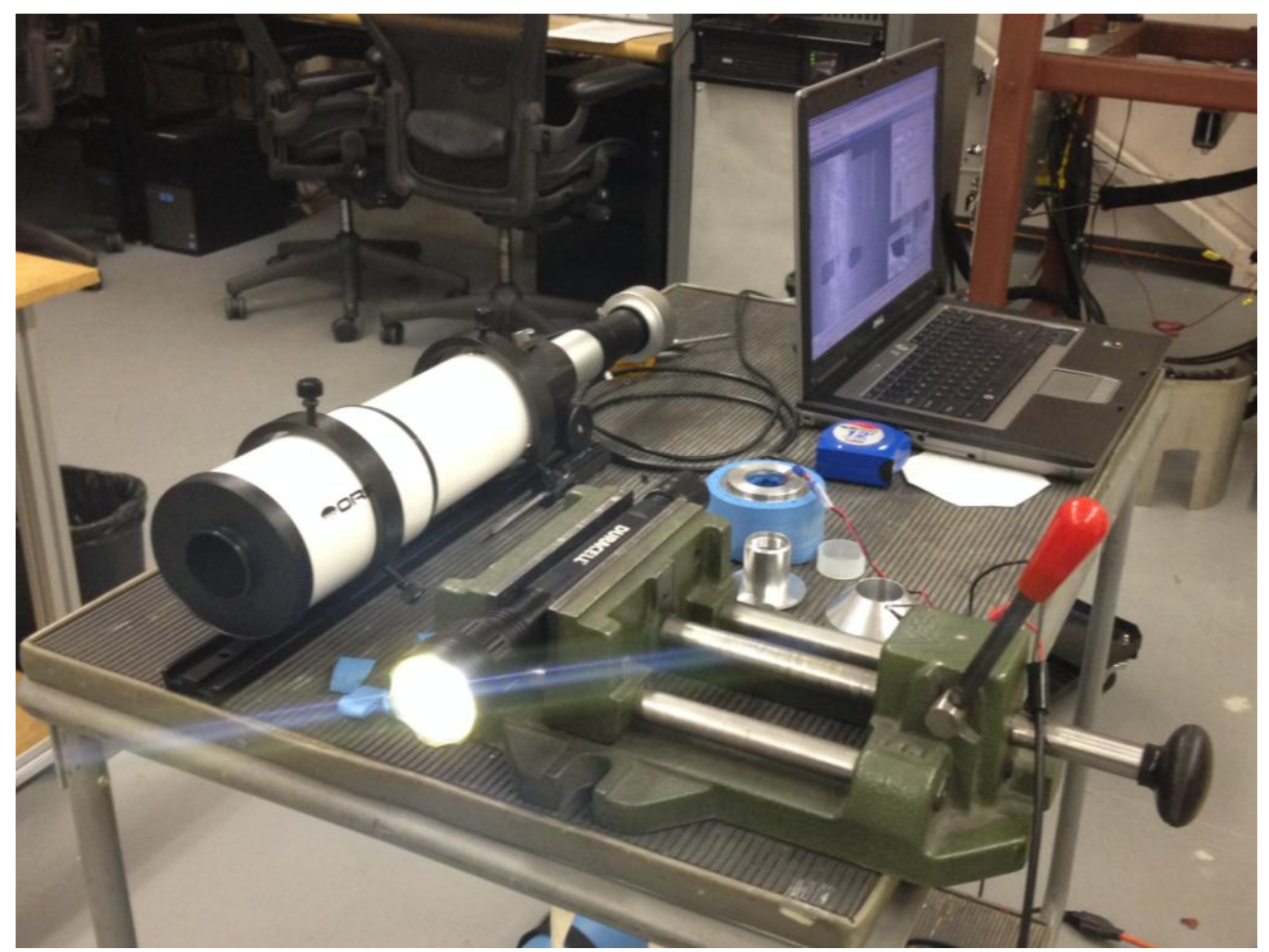

Figure 8. Focused LED light and $43 \mathrm{~mm}$ diameter telescopic lens / camera configuration. 


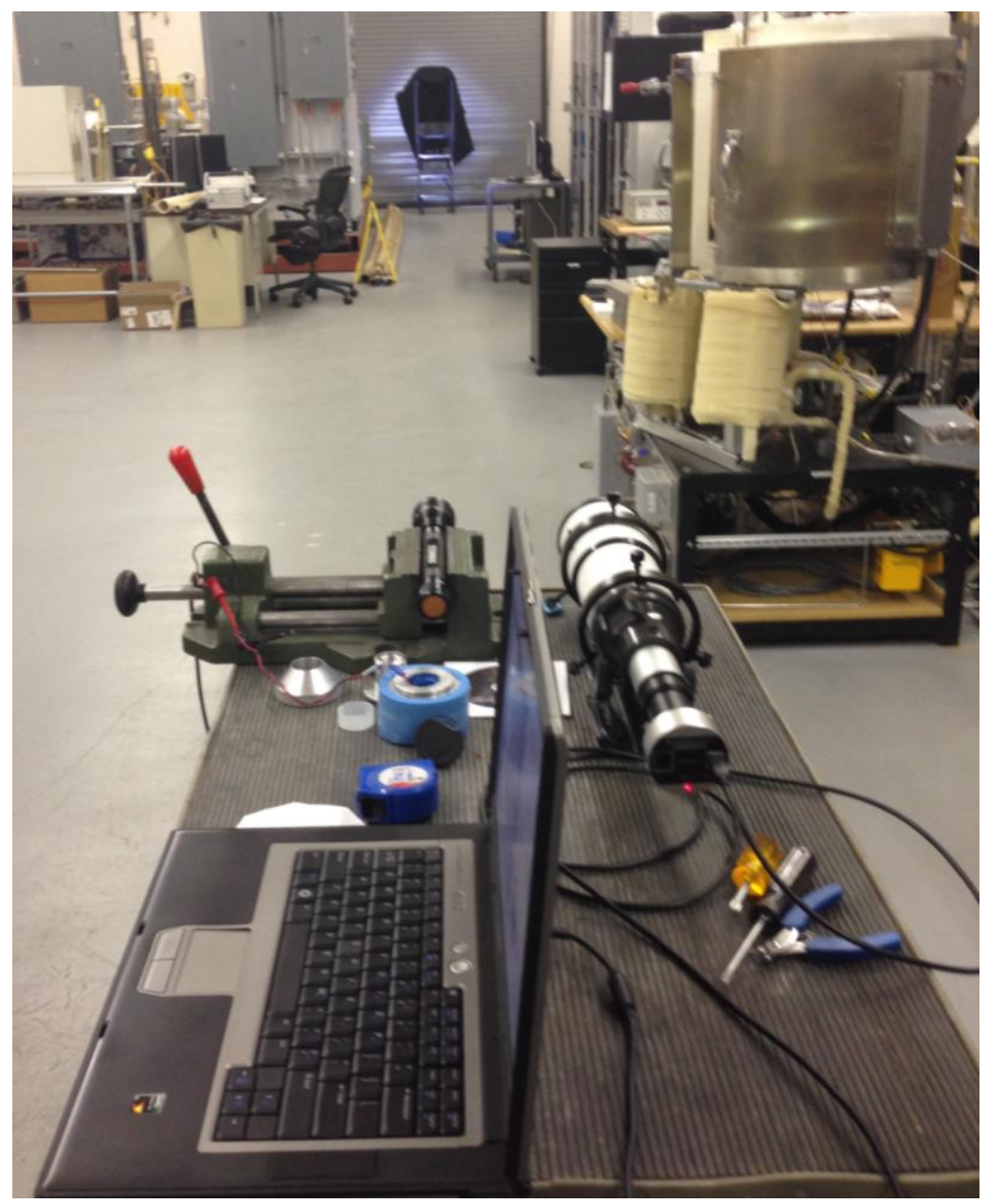

Figure 9. Focused LED “spot” size at $18.4 \mathrm{~m}$ distance.

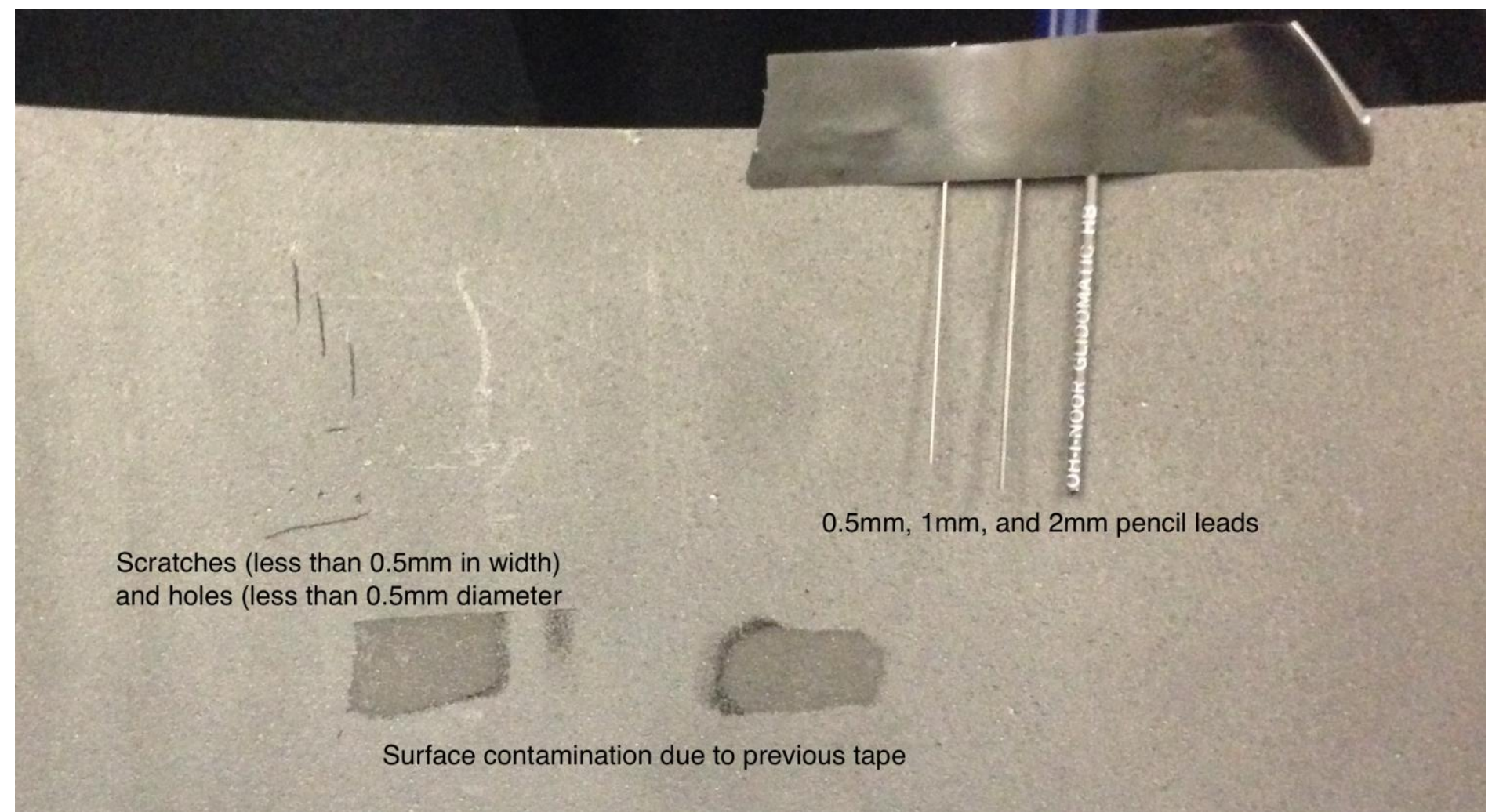

Figure 10. AGR block concave interior surface with target scratches, holes, and pencil leads. 


\section{Scratches}

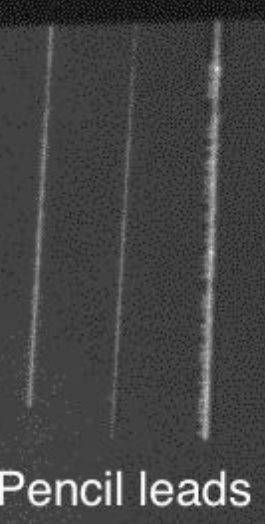

Figure 11. 0.3 second single exposure using $43 \mathrm{~mm}$ diameter, $400 \mathrm{~mm}$ focal length optics at $18.4 \mathrm{~m}$ distance. Only focused beam LED lighting of 1000 lumens was used.

Figure 11 is one sample image acquired using a focused beam LED lighting source. This is a 0.3 sec single exposure (no image stacking was performed) using $43 \mathrm{~mm}$ diameter, $400 \mathrm{~mm}$ focal length optics at $18.4 \mathrm{~m}$ distance. The three different pencil leads are obvious in the image. The scratches and holes are also visible, but are not as obvious. It appears that different types of surface defects will respond differently to the lighting angle of incidence. To explore this somewhat, a flat block of graphite was similarly imaged. Figure 12 is a $0.5 \mathrm{sec}$ single exposure $(43 \mathrm{~mm}$ diameter $400 \mathrm{~mm}$ focal length optics, $18.4 \mathrm{~m}$ distance) using ambient laboratory lighting. Figure 13 is the same field of view, but using focused beam LED lighting. Luckily, the concave interior surface of the AGR block responds better to direct incident lighting, whereas flat graphite surfaces (dependent upon surface conditions) do not respond as well.

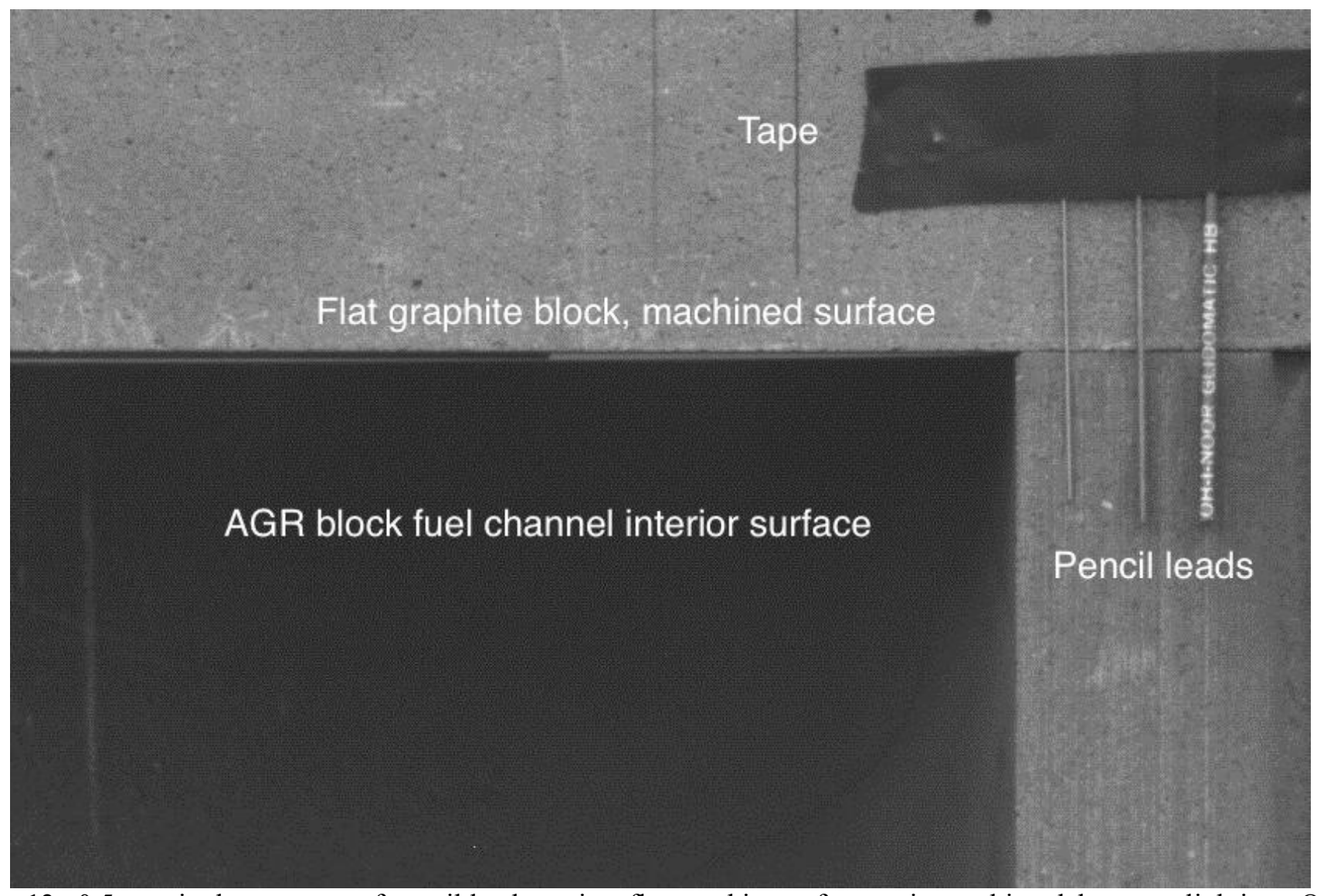

Figure 12. $0.5 \mathrm{sec}$ single exposure of pencil leads against flat graphite surfaces using ambient laboratory lighting. Other parameters same as in Figure 11. 


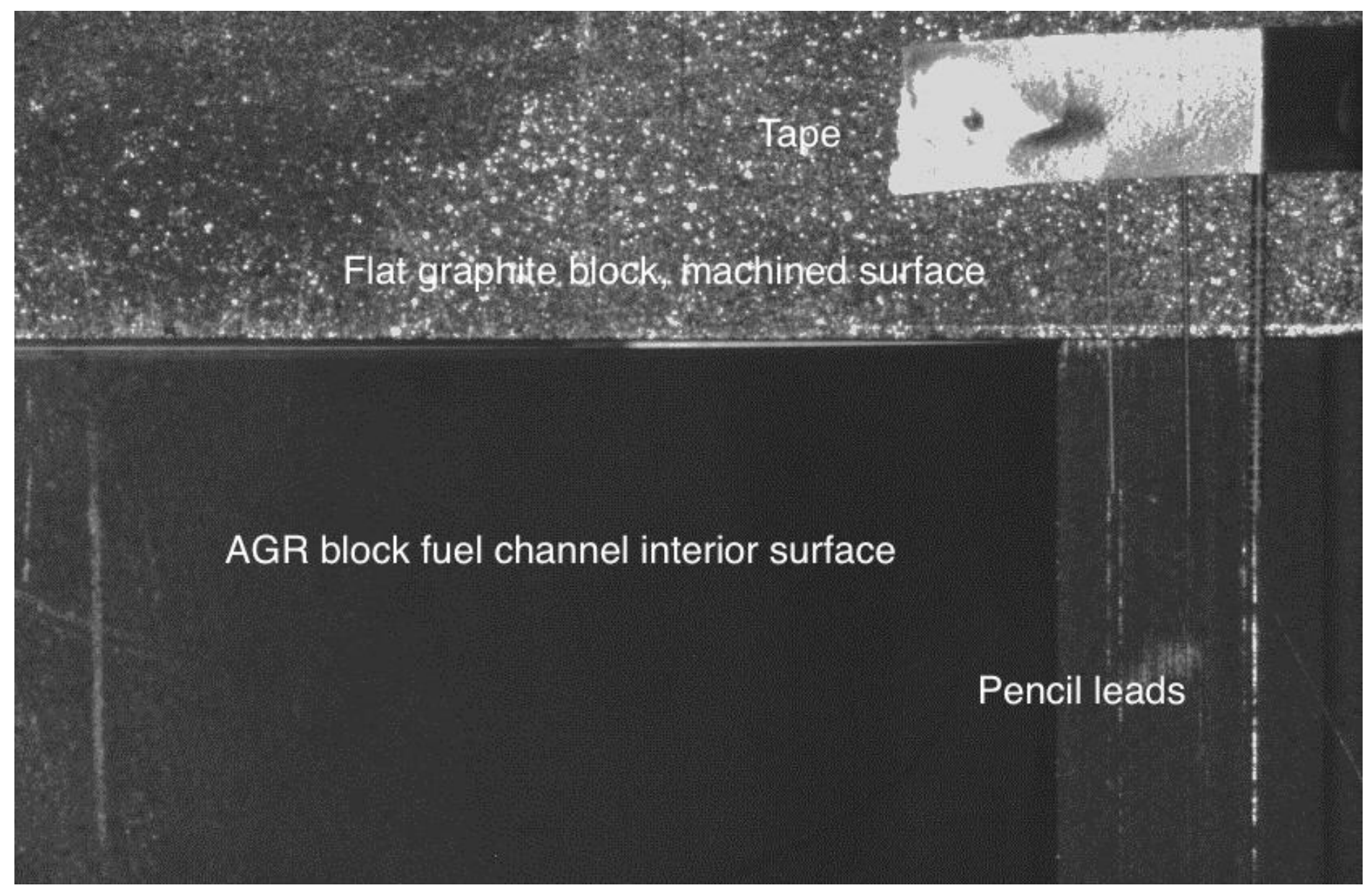

Figure 13. $0.5 \mathrm{sec}$ exposure using focused beam LED lighting of the same field of view as Figure 12. Other parameters are the same as Figure 11.

The INL wanted to test the impact of a right hand mirror on lighting. INL reoriented the sectioned AGR graphite block as depicted in Figure 14. Two right hand mirrors were located within the graphite block. The right mirror is a laser-quality front surface optically flat mirror whereas the left mirror is a curved lower quality mirror used in automobiles for enhancing rear view field of view. The flat mirror shows a reflection of the three pencil leads taped to the AGR graphite fuel channel interior surface. Exposure lengths were set to be $5 \mathrm{sec}$ to reduce the electronic gain of the camera and hence electronic noise. No significant difference was found with changing the image acquisition time from sub second values to 5 seconds. Figure 15 presents a $5 \mathrm{sec}$ single exposure through the flat right hand mirror. The three pencil leads can be seen easily in the reflection of the right hand mirror. The ends of the pencil leads can be seen as well, above the mirror's reflection. There is an additional reflection in the mirror to the right of the pencil leads due to a scar on the graphite block. This test was repeated to see if the graphite block artificial scratches and holes discussed earlier could be detected. Figure 16 is a $5 \mathrm{sec}$ exposure using focused beam LED lighting and $43 \mathrm{~mm}$ diameter $400 \mathrm{~mm}$ focal length telescopic optics, through right hand flat mirror, of these scratched and holes artificially created on graphite surface. The scratches and holes were easily detected.

These tests were repeated for the curved mirror described previously. Figure 17 is a typical 5 sec exposure using focused beam LED lighting and $43 \mathrm{~mm}$ diameter $400 \mathrm{~mm}$ focal length telescopic optics, through right hand curved mirror, of pencil leads against AGR block fuel channel interior surface. The reflection of the pencil leads is not as obvious, but can still be detected. INL believes that the success of employing any curved mirror such as conical mirror will be strongly dependent upon the quality and uniformity of the mirror surface. This is a similar conclusion to that of AMEC in their evaluation of conical right hand mirrors for the FMIT visual inspection tool. 


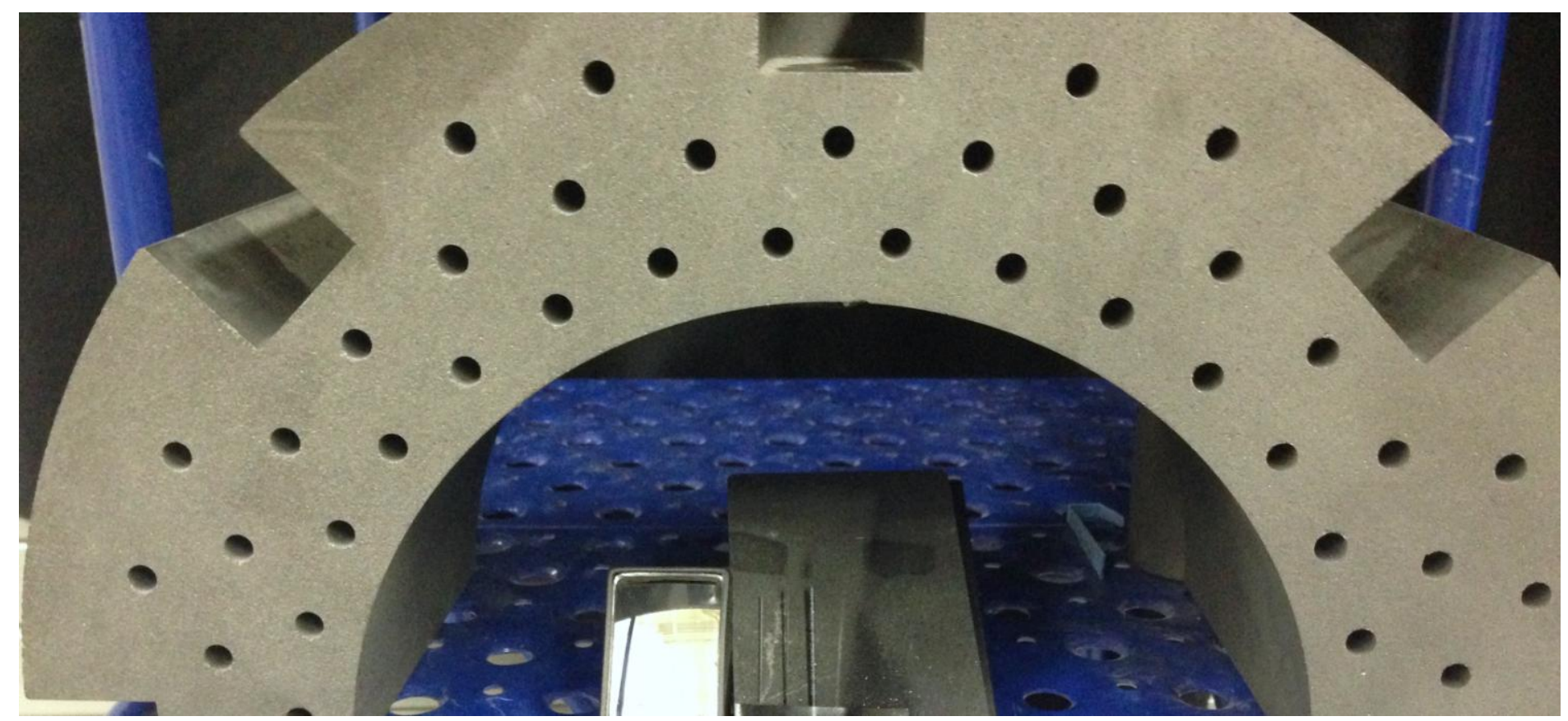

Figure 14. Target orientation for testing image quality using right hand flat and curved mirrors.

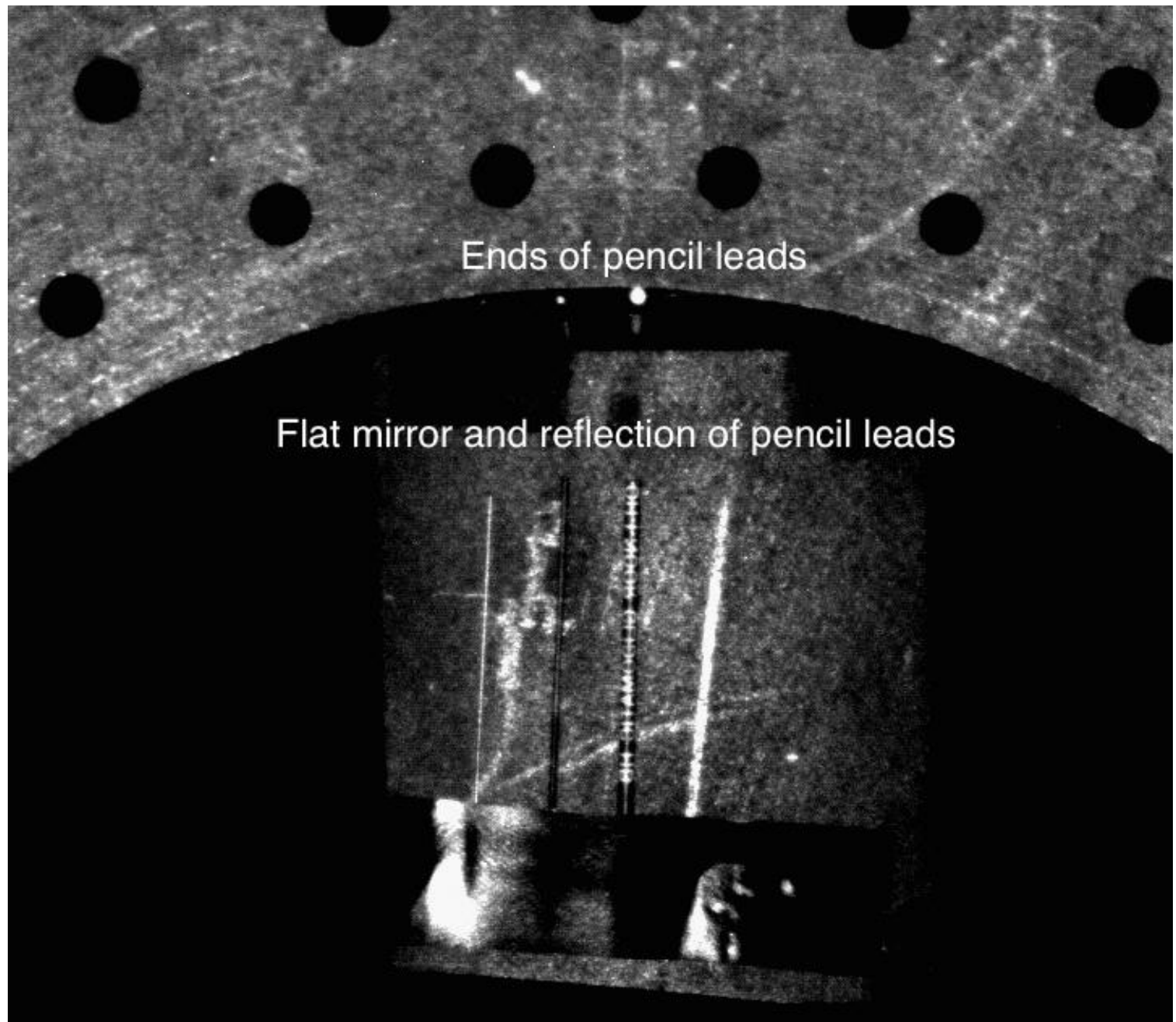

Figure 15. $5 \mathrm{sec}$ exposure using focused beam LED lighting and $43 \mathrm{~mm}$ diameter $400 \mathrm{~mm}$ focal length telescopic optics, through right hand flat mirror. 


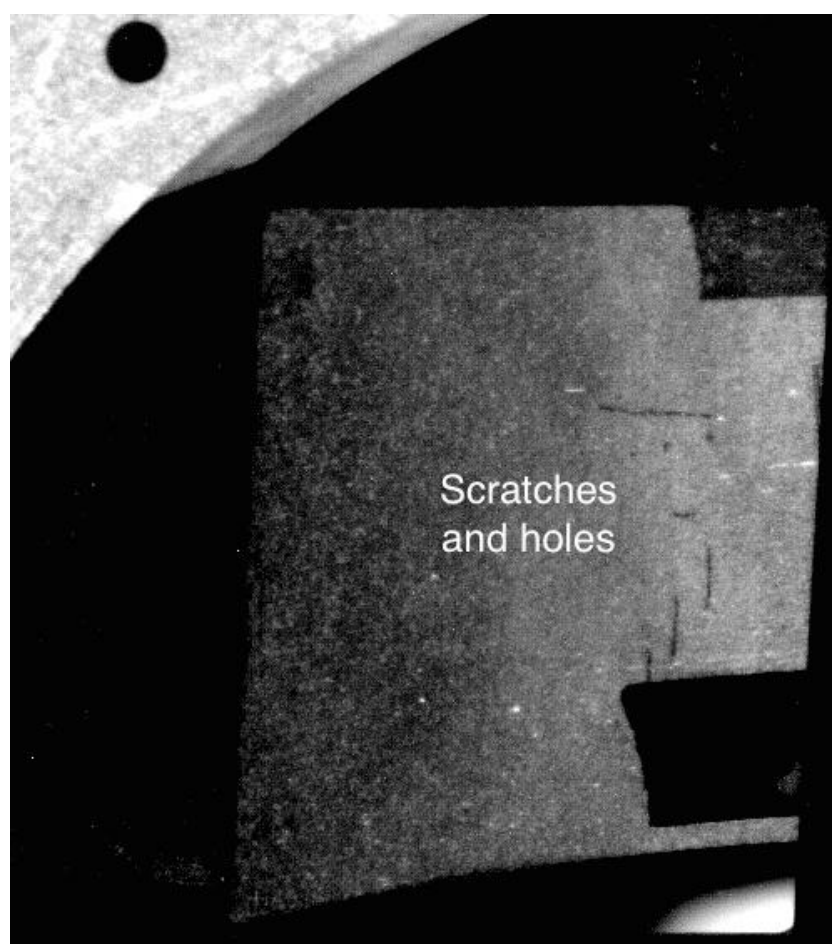

Figure 16. $5 \mathrm{sec}$ exposure using focused beam LED lighting and $43 \mathrm{~mm}$ diameter $400 \mathrm{~mm}$ focal length telescopic optics, through right hand flat mirror, of scratched and holes artificially created on graphite surface.

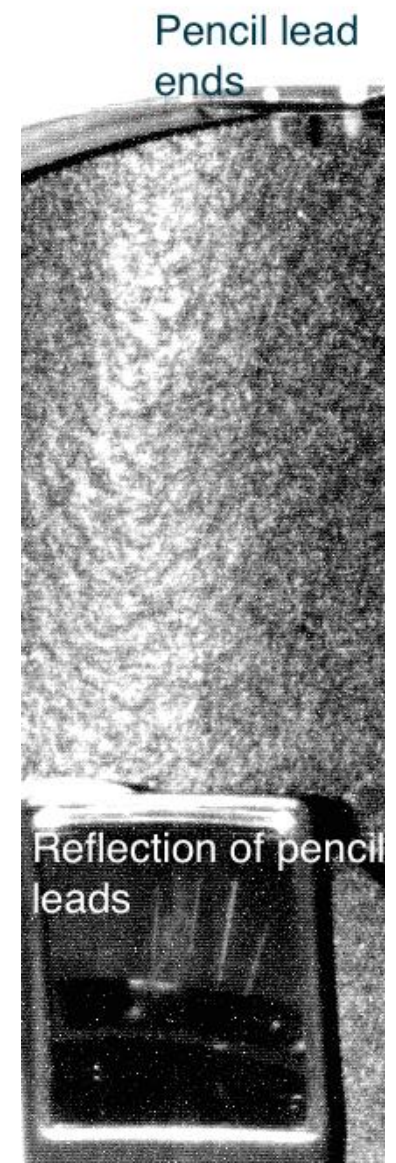

Figure 17. $5 \mathrm{sec}$ exposure using focused beam LED lighting and $43 \mathrm{~mm}$ diameter $400 \mathrm{~mm}$ focal length telescopic optics, through right hand curved mirror, of pencil leads against AGR block fuel channel interior surface. 


\section{Conclusions}

INL calculations and measurements, as well as consideration of AMEC measurement results, lead to the following tentative conclusions. It appears promising that a $43 \mathrm{~mm}$ diameter, $400 \mathrm{ml}$ telescopic lens with a rather low cost CMOSbased digital camera will detect $0.5 \mathrm{~mm}$ width surface features on certain graphite substrates at $20 \mathrm{~m}$ offset distance using a remote (co-located with the telescopic lens) focused beam LED lighting system of about 1000 lumens. It seems that the concave fuel channel interior surface of non-irradiated AGR graphite is compatible with this inspection system whereas other types of graphite surfaces may not be as easily interrogated with this inspection concept. Furthermore, the choice of $43 \mathrm{~mm}$ diameter telescopic optics should fit in the geometric limitations of the FMIT as it is currently understood. Finally, the power requirements of the camera and focused beam LED lighting system and should be compatible with the existing wiring in the FM. The particular camera used by INL is USB-based for data transmission (and power). The FMIT should probably use a Ethernet-based camera for data transmission, due to lead lengths and data transmission signal timing.

\section{Recommendations}

INL suggests that at this point an effort should be made to understand how to package within the constraints of the FMIT the focused beam LED lighting system, telescopic optics, and camera with consideration to radiation shielding, power and data transmission, service and maintenance accessibility, etc. AMEC's studies of a right hand conical mirror, and INL's studies of right hand flat and curved mirrors, stress that the actual mirrors used should be of reasonably high surface and optical quality, while maintaining resistance to radiation browning. Well polished metal conical mirrors should satisfy this requirement. To prove this, INL suggests that some further studies be conducted with high quality conical mirrors in conjunction with a focused beam LED lighting system. Finally, INL suggests some studies be undertaken to quantify how much image quality deterioration may occur due to the AGR inspection environment: vibrations, dust, thermal gradients, etc.

\section{References}

1. INL Technical Report “Advanced Image Systems for High Radiation Environment Applications,” INL/MIS-14-31271, January, 2014.

2. AMEC Technical Report "Fuelling machine deployed inspection tool visual inspection systems", AMEC Report 202718-TER-000003, Issue 01, October, 2014.

3. INL Technical Report "INL Review of Fueling Machine Inspection Tool Development Proposal," INL/EXT-15-34730, March, 2015. 


\section{Addendum}

The INL recently conducted additional tests with an improved camera and optical system. The INL was interested in seeing what improvements, if any, could be had with a 16-bit high sensitivity / low noise cooled camera and better optics. To accomplish this, the INL used an Atik 460EX monochrome CCD camera. This camera uses a Sony ICX694 low noise, high efficiency sensor chip, with $4.54 \mu \mathrm{m}$ pixels. The sensor array is 2750 x 2200 pixels ( 6 megapixels). The sensor has $77 \%$ quantum efficiency in the green wavelength of light. It is also cooled via a Peltier cooler. Cooling increases sensitivity while reducing electronic chip noise. Power requirement for the camera is $12 \mathrm{~V}, 1 \mathrm{~A}$. The camera is shown in Figure 18.

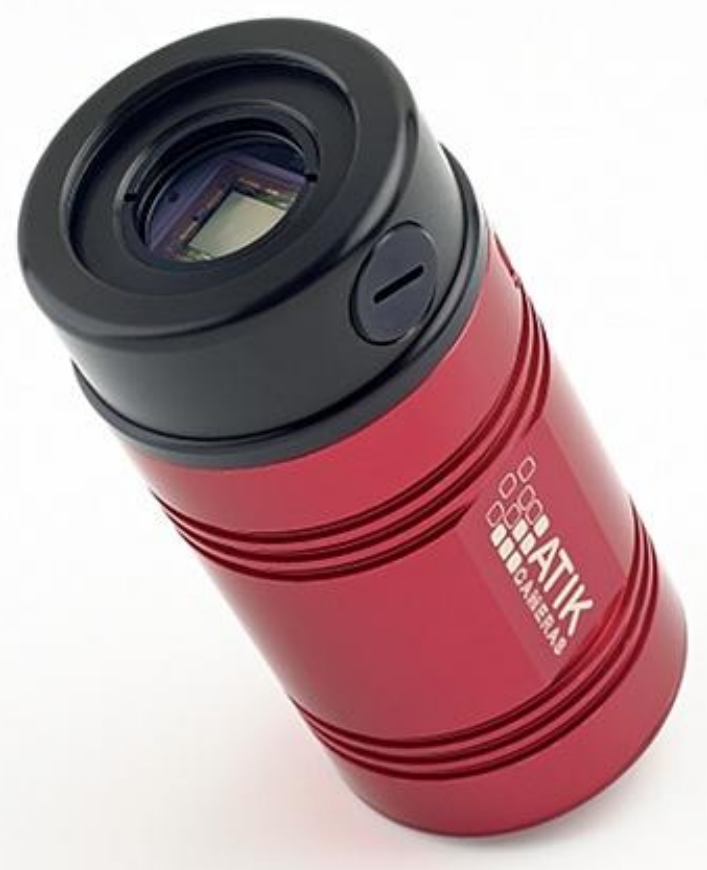

Figure 18. Atik 460EX 6 megapixel low noise high sensitivity cooled monochrome camera used in advanced imaging tests.

It was desired to couple the better camera with better optics as well. The INL utilized a Zeiss Telementor 63mm diameter, $840 \mathrm{~mm}$ focal length refractor with helical focuser. This telescope is widely considered one of the best telescopes ever made in this size range. The new optical imaging system is pictured in Figure 19. It is perhaps interesting to note that this setup begins to resemble a system that could be "packaged" into the EDF UK FMIT system. Also, the combination of camera and LED lighting power requirements remain within the capabilities of the FMIT system. 


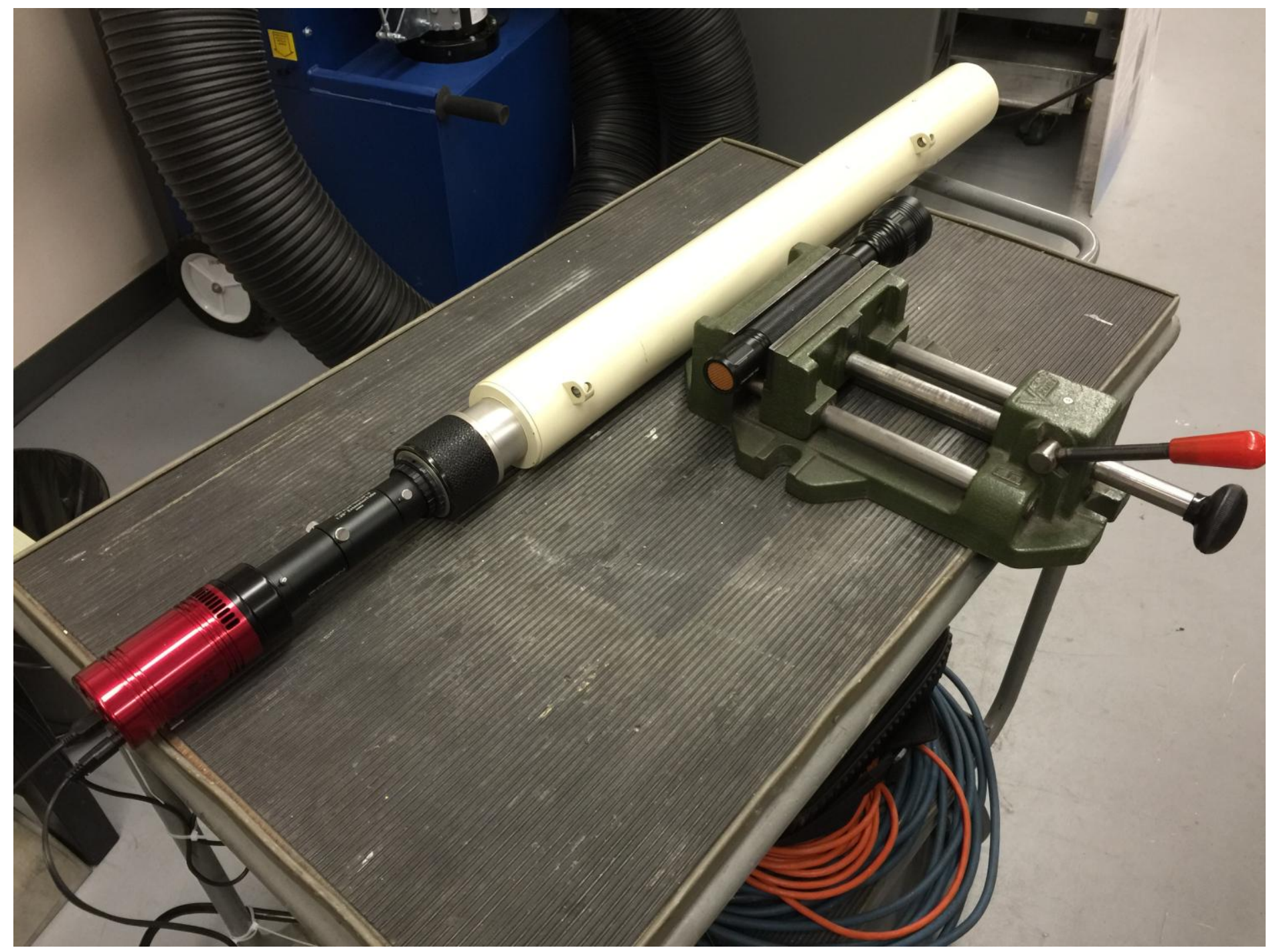

Figure 19. Atik 460EX camera (red) attached to Zeiss Telementor $63 \mathrm{~mm}$ diameter / $840 \mathrm{~mm}$ focal length telescope.

Figure 20 shows the target used for testing this optical configuration. The target uses the same EDF graphite bricks as before, and the same lead rods as before. These lead rods are (from left to right) $0.5 \mathrm{~mm}, 1 \mathrm{~mm}$, and $2 \mathrm{~mm}$ diameter. The $2 \mathrm{~mm}$ lead rod has text on it as well. The block backdrop has scratches as before. The researchers included two stainless steel wires on the target as well. In the left field of view is a $0.5 \mathrm{~mm}$ diameter wire, and in the right field of view is a $0.25 \mathrm{~mm}$ wire. 


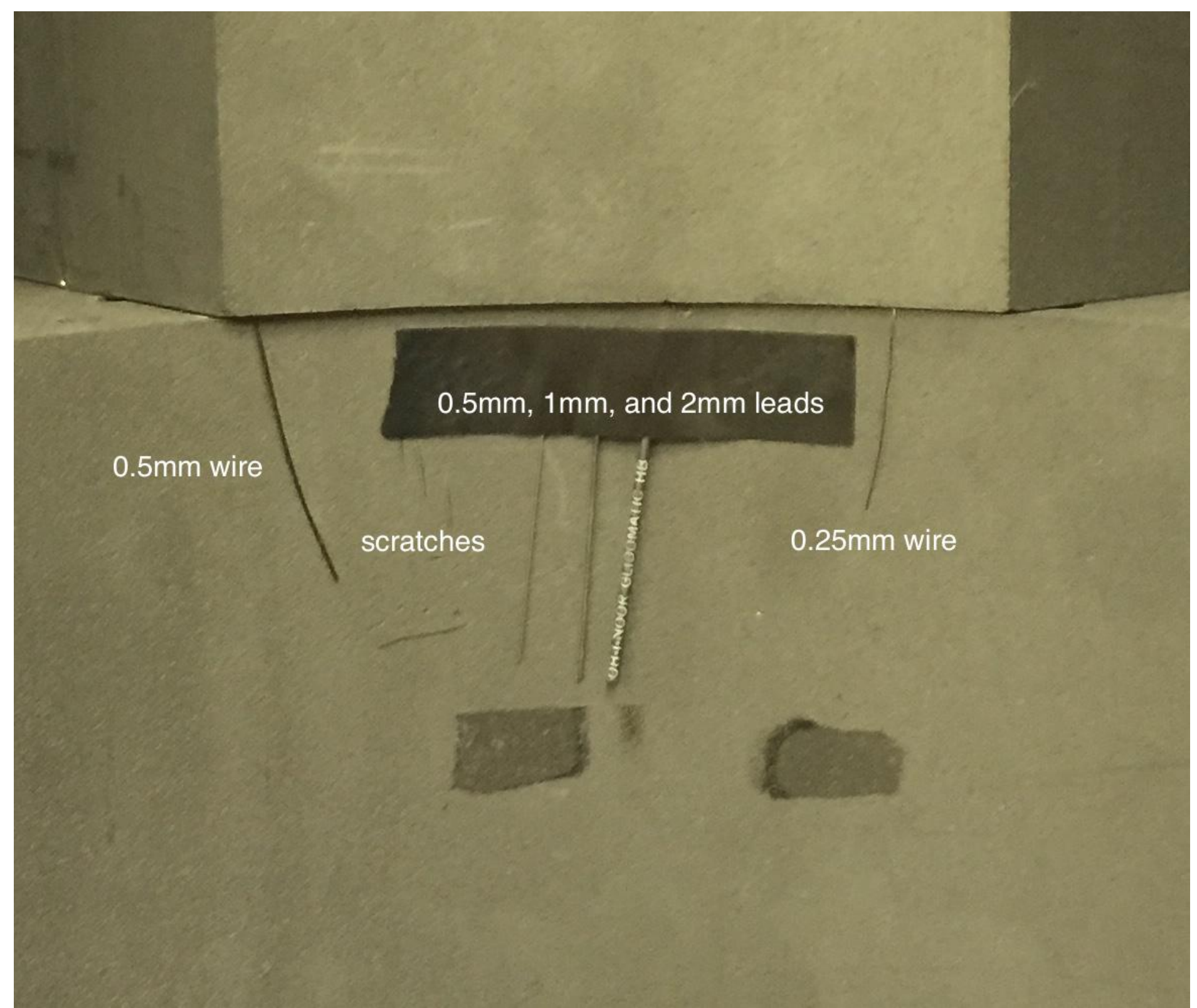

Figure 20. Target used for imaging tests. The target includes the same pencil leads as previously, but also includes a $0.25 \mathrm{~mm}$ (right) and $0.5 \mathrm{~mm}$ (left) diameter wires.

Figures 21, 22, and 23 are sample results from the above-described optical and target configuration. All images were taken from $18.4 \mathrm{~m}$ distance in complete darkness other than the focused LED lighting. Figure 21 is a 5 second exposure, Figure 22 is a 1 second exposure, and Figure 23 is a 0.1 second exposure. The results are quite impressive. The 16 bit resolution of the camera allows much greater gradation of grey scales, resulting in a much smoother image. The added sensitivity and lower noise of the camera allows finer detail and faster exposure times. Even at $0.1 \mathrm{sec}$, all target details are clearly discernable. The possibility of such fast exposure times would greatly reduce the overall time to image a fuel channel. The higher quality optics of the Zeiss telescope allows finer detail and a sharper focus. Finally, the physical size and power requirements of this system are reasonable for packaging into the FMIT graphite inspection tool. It is INL's overall opinion that these latest results prove the viability of this concept for consideration in the FMIT. Of course, the FMIT will be in a much harsher environment (temperature, vibrations, radioactivity, etc.) but addressing the environmental effects were beyond the scope of this proof-of-principle project. 


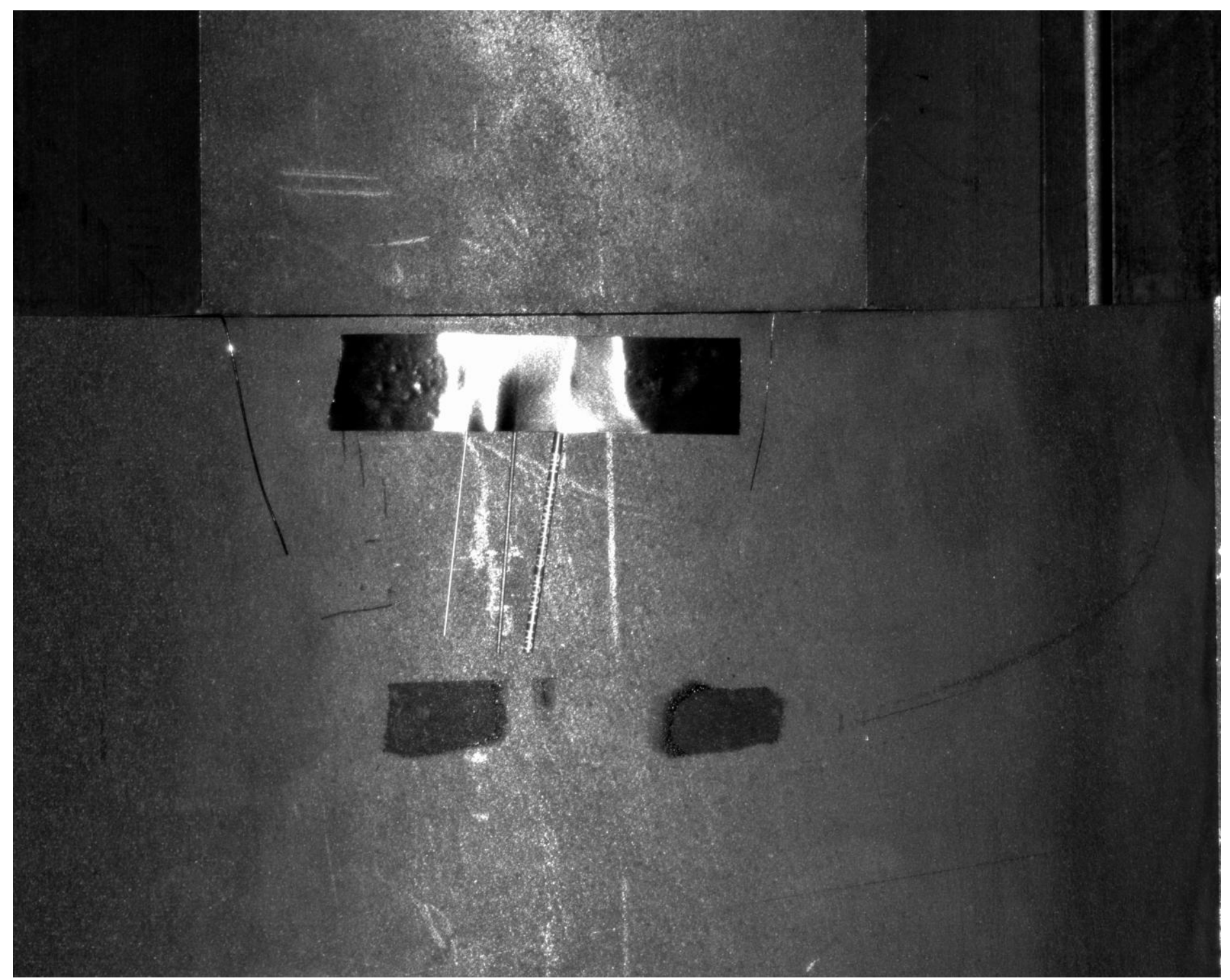

Figure 21. 5 second exposure of target using Atik camera / Zeiss telescope and focused beam LED lighting. 


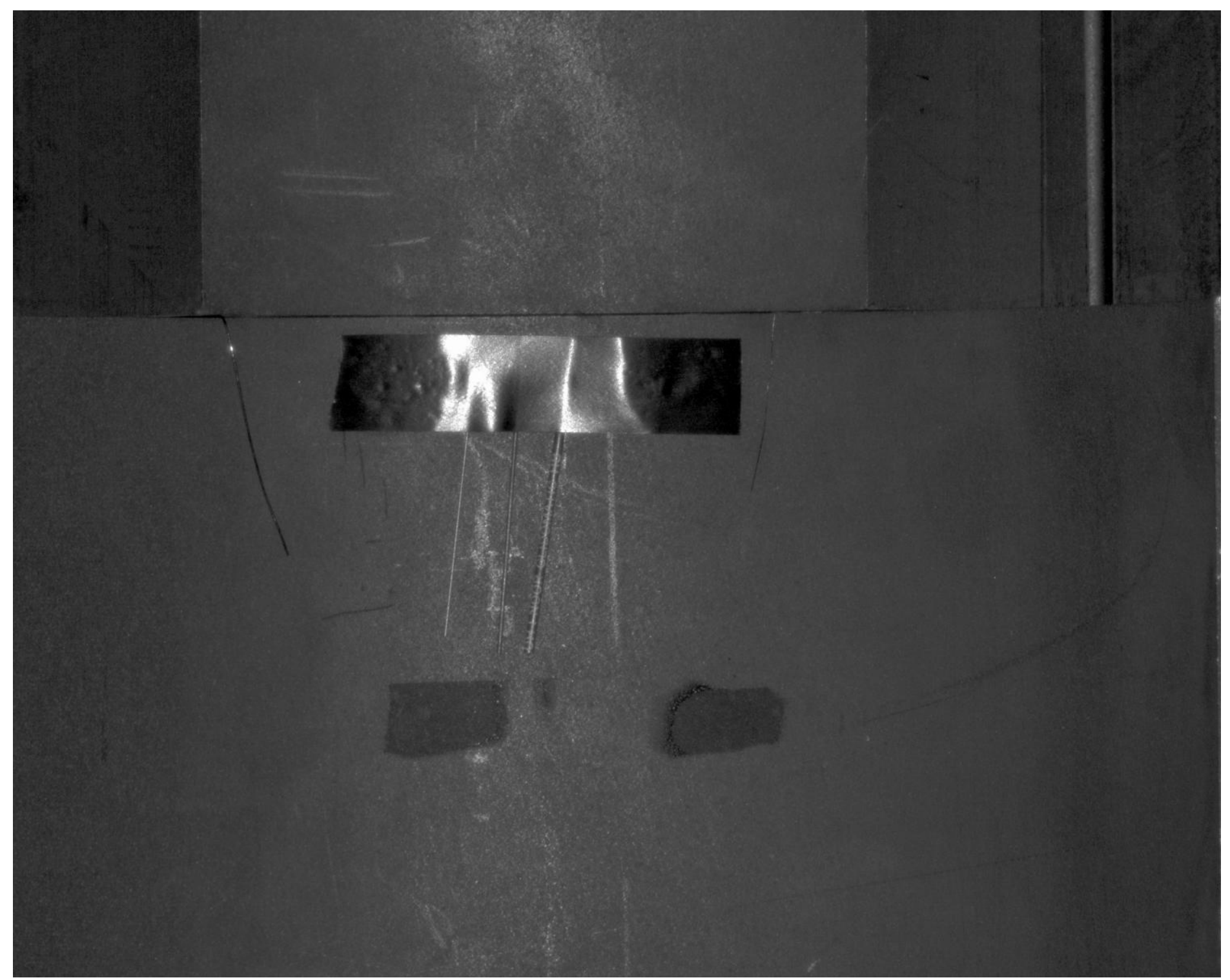

Figure 22. 1 second exposure of target using Atik camera / Zeiss telescope and focused beam LED lighting. 


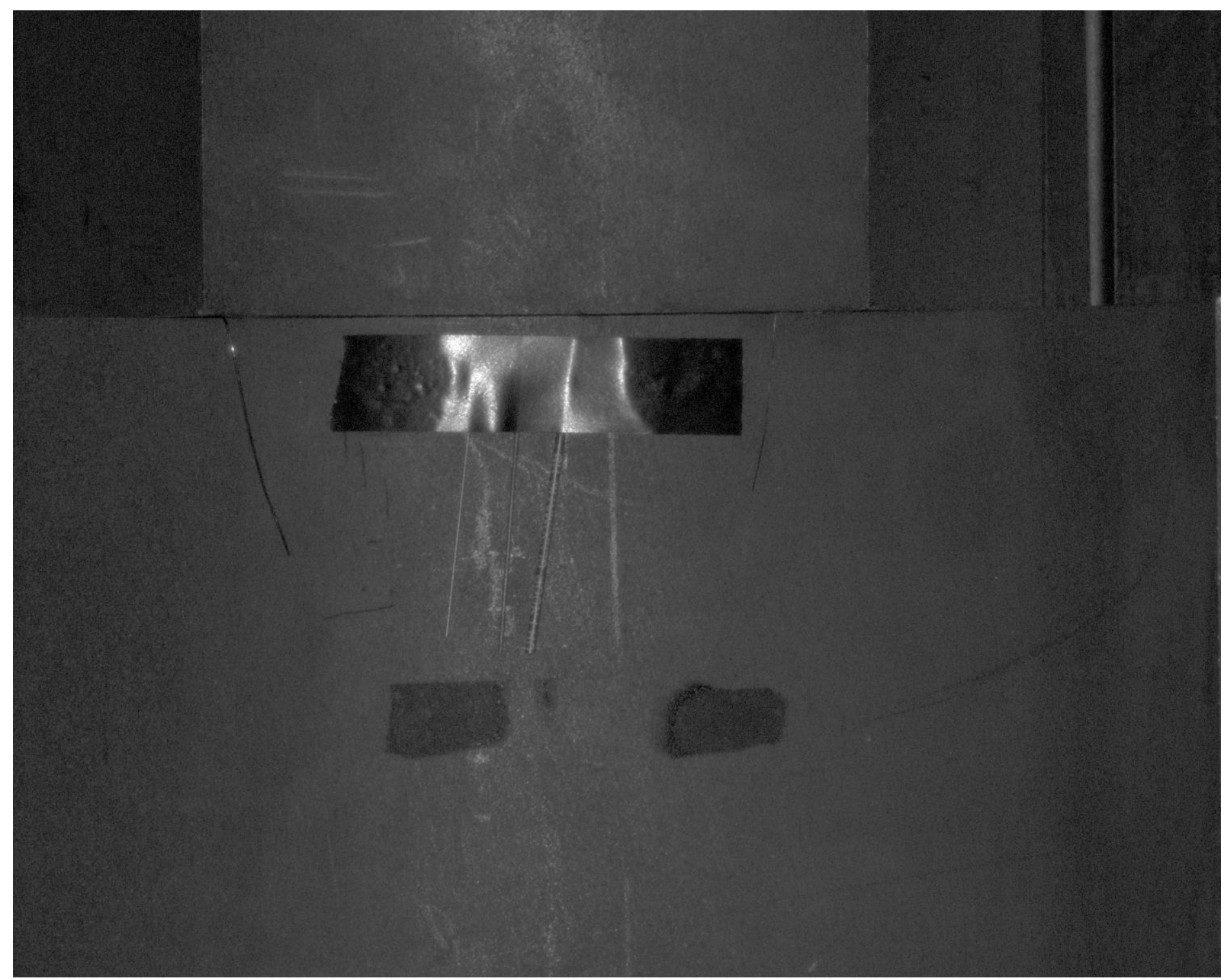

Figure 23. 0.1 second exposure of target using Atik camera / Zeiss telescope and focused beam LED lighting. 